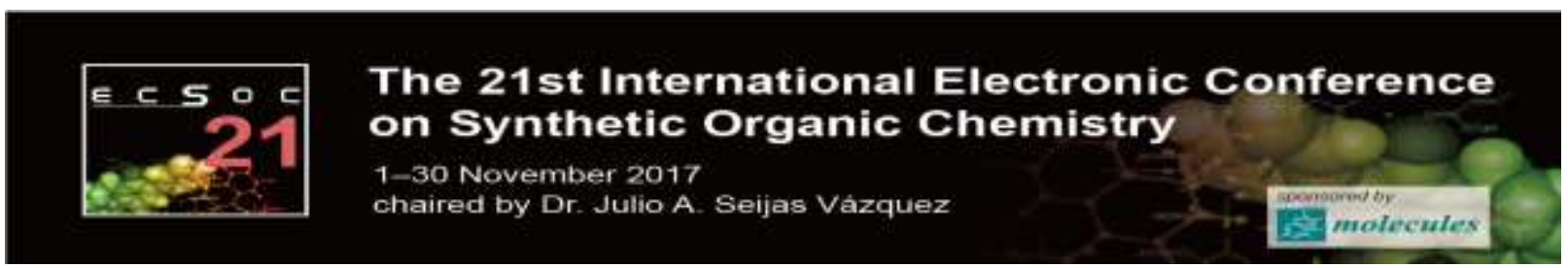

http://sciforum.net/conference/ecsoc-21

\title{
Alkaline functionalized chitosan as highly efficient organocatalyst for the synthesis of 3-indole derivatives
}

\author{
Ehsan Valiey, Mohammad G. Dekamin* and Zahra Alirezvani \\ Pharmaceutical and Biologically-Active Compounds Research Laboratory, Department of Chemistry, Iran University of \\ Science and Technology, Tehran 16846-13114, Iran \\ Tel: +98-21-77240640-50; fax: +98-21-73021584; e-mail: mdekamin@iust.ac.ir
}

\begin{abstract}
A green one-pot multicomponent reaction for the synthesis of 3-indole derivatives was developed via alkaline functionalized chitosan catalyzed condensation between indoles, aldehydes, and malononitrile. The process was carried out in ethanol at room temperature, and the desired products were obtained in good to excellent yields. The salient features of this protocol were environmental friendliness, simplicity of the procedure, ready accessibility of the catalyst, high yield, and mild reaction conditions.
\end{abstract}

\section{Introduction}

Multicomponent reactions (MCRs), have gained much attention in synthetic organic chemistry or significant advantages of atom economy, time-saving, inherent exploratory power and minimization of chemical waste. Moreover, this strategy has proved to be a particularly valuable and efficient methodology in the section of synthetic chemistry. The indole is probably one of the most ubiquitous moieties in nature. A vast number of natural and synthetic indole derivatives 
have been found a respected value in pharmaceutical and medical applications since they are able to bind with high affinity to many receptors. Among them, 3-substituted indole derivatives have attracted much attention because they exist in many natural products, and their simple structure with broad scope of potent biological activities.[1,2]

In continuation of our efforts in the synthesis and application of new heterogenous catalyst for organic transformations[3-6], Herein, we report our results for a facile and convenient synthesis of 3-indole derivatives using alkaline functionalized chitosan (1) as a highly efficient metal free organocatalyst

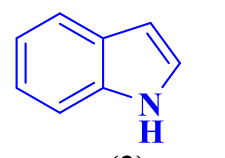

(2)

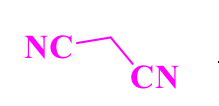

(3)

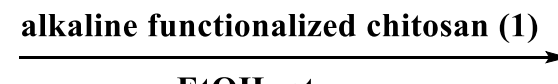

EtOH, r.t.

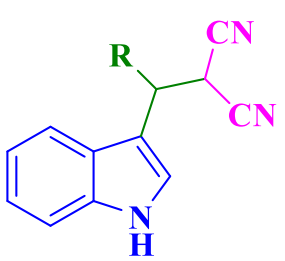

(5)

Scheme 2. Synthesis of 3-indole derivatives catalyzed by alkaline functionalized chitosan (1)

\section{Experimental section}

\subsection{General}

\section{Reagents and Apparatus}

High-purity chemical reagents were purchased from Merck. All reactions and the purity of the products were monitored by thin-layer chromatography (TLC) using aluminum plates coated with silica gel F254 plates (Merck) using ethyl acetate and n-hexane as eluents. The spots were 
detected either under UV light or by placing in an iodine chamber. Melting points were determined in open capillaries using an Electrothermal 9100.

General procedure for the synthesis of bis(indolyl)methane derivatives (4a-f)

In a round-bottomed flask, indole $(\mathbf{2}, 1 \mathrm{mmol})$, malononitrile $(\mathbf{3}, 1 \mathrm{mmol})$, various aldehydes $(\mathbf{4}, 1$ mmol) and $5 \mathrm{mg}$ alkaline functionalized chitosan (1) were added to EtOH 96\% (2 mL). The obtained mixture was stirred at room temperature. After completion of the reaction monitored by TLC (eluent: 25\% v/v EtOAc/n-hexane), EtOH 96\% (3 mL) was added and the obtained mixture filtered off to separate the catalyst 1 . The filtrate was evaporated to dryness under reduced pressure.

\section{Results and discussion}

The catalytic facility of the alkaline functionalized chitosan (1) was evaluated in catalyzing a reaction for the efficient synthesis of 3 -indole derivatives by condensing indole, various aldehydes and malononitrile in $\mathrm{EtOH}$ at room temperature. The results were evaluated qualitatively through TLC. It was found that the quantitative yield can be achieved when the reaction was carried out in the presence of $0.005 \mathrm{~g}$ catalyst. The results are summarized in scheme 3 . The alkaline functionalized chitosan (1) were easily separated via filtrations and the recovered catalyst was reused for at least 7 runs without significant degradation in catalytic activity and performance. 
<smiles>N#CC(C#N)C(c1ccccc1)c1c[nH]c2ccccc12</smiles>

$4 a$<smiles>COc1ccc(C(c2c[nH]c3ccccc23)C(C#N)C#N)cc1</smiles>

4d<smiles>N#CC(C#N)C(c1ccc(Cl)cc1)c1c[nH]c2ccccc12</smiles>

4b<smiles>N#CC(C#N)C(c1ccc(Cl)cc1Cl)c1c[nH]c2ccccc12</smiles>

$4 e$<smiles>COc1ccc(C(c2c[nH]c3ccccc23)C(C#N)C#N)cc1</smiles>

4c<smiles>N#CC(C#N)C(c1ccccc1Cl)c1c[nH]c2ccccc12</smiles>

$4 f$

Scheme 3. Scope of 3-indole derivatives (4a-f)

\section{Conclusion}

In summary, a new eco-friendly method for the efficient synthesis of indole derivatives, catalyzed by alkaline functionalized chitosan as a new recyclable heterogeneous catalyst under room temperature conditions was introduced. Furthermore, the present method offers several advantages such as mild conditions, quantitative yields of products in relatively short reaction times, elimination of toxic solvent, clean reaction profile and simple work-up procedure, and savings in energy consumption that makes this method an instrumental alternative to the previous methodologies for the scale-up of these one-pot three-component reactions 


\section{Acknowledgements}

We are grateful for the financial support from The Research Council of Iran University of Science and Technology (IUST), Tehran, Iran (Grant no. 160/8034).

\section{References}

[1] Y.-H. He, J.-F. Cao, R. Li, Y. Xiang, D.-C. Yang, Z. Guan, L-Proline-catalyzed multicomponent synthesis of 3-indole derivatives, Tetrahedron 71(49) (2015) 9299-9306.

[2] M. Shiri, Indoles in multicomponent processes (MCPs), Chemical reviews 112(6) (2012) 3508-3549.

[3] A. Azad, M.G. Dekamin, S. Afshar, A. Tadjarodi, A. Mollahosseini, Activation of hexamethyldisilazane (HMDS) by $\mathrm{TiO} 2$ nanoparticles for protection of alcohols and phenols: the effect of the catalyst phase on catalytic activity, Research on Chemical Intermediates 1-13.

[4] M.G. Dekamin, F. Mehdipoor, A. Yaghoubi, 1, 3, 5-Tris (2-hydroxyethyl) isocyanurate functionalized graphene oxide: a novel and efficient nanocatalyst for the one-pot synthesis of 3 , 4-dihydropyrimidin-2 (1H)-ones, New Journal of Chemistry (2017).

[5] A. Yaghoubi, M.G. Dekamin, B. Karimi, Propylsulfonic Acid-Anchored Isocyanurate-Based Periodic Mesoporous Organosilica (PMO-ICS-PrSO3H): A Highly Efficient and Recoverable Nanoporous Catalyst for the One-Pot Synthesis of Substituted Polyhydroquinolines, Catalysis Letters (2017) 1-8. 
[6] M.G. Dekamin, S. Ilkhanizadeh, Z. Latifidoost, H. Daemi, Z. Karimi, M. Barikani, Alginic acid: a highly efficient renewable and heterogeneous biopolymeric catalyst for one-pot synthesis of the Hantzsch 1, 4-dihydropyridines, RSC Advances 4(100) (2014) 56658-56664. 